\title{
SUBMODULES OF THE HARDY MODULE OVER POLYDISC
}

\author{
JAYDEB SARKAR \\ Dedicated to Ronald G. Douglas on the occasion of his 75th birthday
}

\begin{abstract}
We say that a submodule $\mathcal{S}$ of $H^{2}\left(\mathbb{D}^{n}\right)(n>1)$ is co-doubly commuting if the quotient module $H^{2}\left(\mathbb{D}^{n}\right) / \mathcal{S}$ is doubly commuting. We show that a co-doubly commuting submodule of $H^{2}\left(\mathbb{D}^{n}\right)$ is essentially doubly commuting if and only if the corresponding one variable inner functions are finite Blaschke products or that $n=2$. In particular, a co-doubly commuting submodule $\mathcal{S}$ of $H^{2}\left(\mathbb{D}^{n}\right)$ is essentially doubly commuting if and only if $n=2$ or that $\mathcal{S}$ is of finite co-dimension. We obtain an explicit representation of the Beurling-LaxHalmos inner functions for those submodules of $H_{H^{2}\left(\mathbb{D}^{n-1}\right)}^{2}(\mathbb{D})$ which are co-doubly commuting submodules of $H^{2}\left(\mathbb{D}^{n}\right)$. Finally, we prove that a pair of co-doubly commuting submodules of $H^{2}\left(\mathbb{D}^{n}\right)$ are unitarily equivalent if and only if they are equal.
\end{abstract}

\section{INTRODUCTION}

Let $\left\{T_{1}, \ldots, T_{n}\right\}$ be a set of $n$ commuting bounded linear operators on a separable Hilbert space $\mathcal{H}$. Then we can turn the $n$-tuple $\left(T_{1}, \ldots, T_{n}\right)$ on $\mathcal{H}$ into a Hilbert module [16] $\mathcal{H}$ over $\mathbb{C}[\boldsymbol{z}]:=\mathbb{C}\left[z_{1}, \ldots, z_{n}\right]$, the ring of polynomials, as follows:

$$
\mathbb{C}[\boldsymbol{z}] \times \mathcal{H} \rightarrow \mathcal{H}, \quad(p, h) \mapsto p\left(T_{1}, \ldots, T_{n}\right) h,
$$

for all $p \in \mathbb{C}[\boldsymbol{z}]$ and $h \in \mathcal{H}$. The module multiplication operators by the coordinate functions on $\mathcal{H}$ are defined by $M_{z_{i}} h=z_{i}\left(T_{1}, \ldots, T_{n}\right) h=T_{i} h$, for all $h \in \mathcal{H}$ and $i=1, \ldots, n$. Therefore, a Hilbert module is uniquely determined by the underlying commuting operators via the module multiplication operators by the coordinate functions and vice versa. Let $\mathcal{S}, \mathcal{Q} \subseteq \mathcal{H}$ be closed subspaces of $\mathcal{H}$. Then $\mathcal{S}(\mathcal{Q})$ is said to be a submodule (quotient module) of $\mathcal{H}$ if $M_{z_{i}} \mathcal{S} \subseteq \mathcal{S}\left(M_{z_{i}}^{*} \mathcal{Q} \subseteq \mathcal{Q}\right)$ for all $i=1, \ldots, n$. Note that a closed subspace $\mathcal{Q}$ is a quotient module of $\mathcal{H}$ if and only if $\mathcal{Q}^{\perp} \cong \mathcal{H} / \mathcal{Q}$ is submodule of $\mathcal{H}$.

The Hardy module $H^{2}\left(\mathbb{D}^{n}\right)$ over the polydisc is the Hardy space $H^{2}\left(\mathbb{D}^{n}\right)$ (cf. [17] and [27]), the closure of $\mathbb{C}[\boldsymbol{z}]$ in $L^{2}\left(\mathbb{T}^{n}\right)$, with the standard multiplication operators by the coordinate functions $z_{i}(1 \leq i \leq n)$ on $H^{2}\left(\mathbb{D}^{n}\right)$ as the module maps.

The module multiplication operators on a submodule $\mathcal{S}$ and a quotient module $\mathcal{Q}$ of a Hilbert module $\mathcal{H}$ are given by the restrictions $\left(R_{z_{1}}, \ldots, R_{z_{n}}\right)$ and the compressions $\left(C_{z_{1}}, \ldots, C_{z_{n}}\right)$ of the module multiplications of $\mathcal{H}$, respectively. That is,

$$
R_{z_{i}}=\left.M_{z_{i}}\right|_{\mathcal{S}} \text { and } C_{z_{i}}=\left.P_{\mathcal{Q}} M_{z_{i}}\right|_{\mathcal{Q}}
$$

2010 Mathematics Subject Classification. 47A13, 47A15, 47A20, 47A45, 47A80, 46E20, 30H10.

Key words and phrases. Hilbert modules, invariant subspaces, Beurling-Lax-Halmos theorem, essentially normal Hilbert modules, rigidity of submodules. 
for all $i=1, \ldots, n$. Here, for a given closed subspace $\mathcal{M}$ of a Hilbert space $\mathcal{K}$, we denote the orthogonal projection of $\mathcal{K}$ onto $\mathcal{M}$ by $P_{\mathcal{M}}$.

A quotient module $\mathcal{Q}$ of a Hilbert module $\mathcal{H}$ over $\mathbb{C}[\boldsymbol{z}](n \geq 2)$ is said to be doubly commuting quotient module if

$$
C_{z_{i}} C_{z_{j}}^{*}=C_{z_{j}}^{*} C_{z_{i}}
$$

for all $1 \leq i<j \leq n$. Also a submodule $\mathcal{S}$ of $\mathcal{H}$ is said to be co-doubly commuting submodule of $\mathcal{H}$ if $\mathcal{H} / \mathcal{S}$ is doubly commuting quotient module of $\mathcal{H}$ (see [28]).

Finally, we recall that a Hilbert module $\mathcal{H}$ over $\mathbb{C}[\boldsymbol{z}]$ is said to be essentially doubly commuting if the cross-commutators

$$
\left[M_{z_{i}}^{*}, M_{z_{j}}\right] \in \mathcal{K}(\mathcal{H})
$$

for all $1 \leq i<j \leq n$, where $\mathcal{K}(\mathcal{H})$ is the ideal of all compact operators on $\mathcal{H}$. We say that $\mathcal{H}$ is essentially normal if $\left[M_{z_{i}}^{*}, M_{z_{j}}\right] \in \mathcal{K}(\mathcal{H})$ for all $1 \leq i, j \leq n$.

Natural examples of essentially normal Hilbert modules are the Drury-Arveson module $H_{n}^{2}$, the Hardy module $H^{2}\left(\mathbb{B}^{n}\right)$ and the Bergman module $L_{a}^{2}\left(\mathbb{B}^{n}\right)$ over the unit ball $\mathbb{B}^{n}$ (cf. [10], [4], [3]). On the other hand, the Hardy module $H^{2}\left(\mathbb{D}^{n}\right)$ over $\mathbb{D}^{n}$ with $n \geq 2$ is not essentially normal. However, a simple calculation reveals that $H^{2}\left(\mathbb{D}^{n}\right)$ is doubly commuting and, in particular, essentially doubly commuting. Therefore, a natural approach to measure a submodule (quotient module) of the Hardy module $H^{2}\left(\mathbb{D}^{n}\right)$ from being "small" is to consider the cross commutators $\left[R_{z_{i}}^{*}, R_{z_{j}}\right]\left(\left[C_{z_{i}}^{*}, C_{z_{j}}\right]\right)$ for all $1 \leq i<j \leq n$ instead of all possible commutators.

Before proceeding further, let us recall the Beurling-Lax-Halmos theorem concerning submodules of vector-valued Hardy modules over $\mathbb{D}$ (cf. [23]).

Given a separable Hilbert space $\mathcal{E}$ we shall denote by $H_{\mathcal{E}}^{2}(\mathbb{D})$ the $\mathcal{E}$-valued Hardy module (see [23]). Note that by virtue of the unitary module map $U: H_{\mathcal{E}}^{2}(\mathbb{D}) \rightarrow H^{2}(\mathbb{D}) \otimes \mathcal{E}$ defined by

$$
z^{m} \eta \mapsto z^{m} \otimes \eta, \quad(\eta \in \mathcal{E}, m \in \mathbb{N})
$$

we can identify the vector-valued Hardy module $H_{\mathcal{E}}^{2}(\mathbb{D})$ with $H^{2}(\mathbb{D}) \otimes \mathcal{E}$.

TheOREM 1.1. (Beurling-Lax-Halmos) Let $\mathcal{E}$ be a Hilbert space and $\mathcal{S}$ be a non-trivial closed subspace of the Hardy module $H_{\mathcal{E}}^{2}(\mathbb{D})$. Then $\mathcal{S}$ is a submodule of $H_{\mathcal{E}}^{2}(\mathbb{D})$ if and only if

$$
\mathcal{S}=\Theta H_{\mathcal{F}}^{2}(\mathbb{D})
$$

where $\Theta \in H_{\mathcal{L}(\mathcal{F}, \mathcal{E})}^{\infty}(\mathbb{D})$ is an inner function and $\mathcal{F}$ is an adequate Hilbert space with dimension less than or equal to the dimension of $\mathcal{E}$. Moreover, $\Theta$ is unique up to a unitary constant right factor, that is, if $\mathcal{S}=\tilde{\Theta} H_{\tilde{\mathcal{F}}}^{2}(\mathbb{D})$ for some Hilbert space $\tilde{\mathcal{F}}$ and inner function $\tilde{\Theta} \in H_{\mathcal{L}(\tilde{\mathcal{F}}, \mathcal{E})}^{\infty}(\mathbb{D})$, then $\Theta=\tilde{\Theta} W$ where $W$ is a unitary operator in $\mathcal{L}(\mathcal{F}, \tilde{\mathcal{F}})$.

Now we formulate some general problems concerning submodules of the Hardy module $H^{2}\left(\mathbb{D}^{n}\right)(n \geq 2)$.

Question 1. (Essentially doubly commuting submodules) How to characterize essentially doubly commuting submodules of the Hardy modules $H^{2}\left(\mathbb{D}^{n}\right)$ ? 
Let $\mathcal{S} \neq\{0\}$ be a closed subspace of $H_{H^{2}\left(\mathbb{D}^{n-1}\right)}^{2}(\mathbb{D})$. By Beurling-Lax-Halmos theorem, Theorem 1.1, that $\mathcal{S}$ is a submodule of $H_{H^{2}\left(\mathbb{D}^{n-1}\right)}^{2}(\mathbb{D})$ if and only if $\mathcal{S}=\Theta H_{\mathcal{E}_{*}}^{2}(\mathbb{D})$, for some closed subspace $\mathcal{E}_{*} \subseteq H^{2}\left(\mathbb{D}^{n-1}\right)$ and inner function $\Theta \in H_{\mathcal{L}\left(\mathcal{E}_{*}, H^{2}\left(\mathbb{D}^{n-1}\right)\right)}^{\infty}(\mathbb{D})$.

QueSTION 2. (Beurling-Lax-Halmos representations) For which closed subspace $\mathcal{E}_{*} \subseteq H^{2}\left(\mathbb{D}^{n-1}\right)$ and inner function $\Theta \in H_{\mathcal{L}\left(\mathcal{E}_{*}, H^{2}\left(\mathbb{D}^{n-1}\right)\right)}^{\infty}(\mathbb{D})$ the submodule $\Theta H_{\mathcal{E}_{*}}^{2}(\mathbb{D})$ of $H_{H^{2}\left(\mathbb{D}^{n-1}\right)}^{2}(\mathbb{D})$, realized as a subspace of $H^{2}\left(\mathbb{D}^{n}\right)$, is a submodule of $H^{2}\left(\mathbb{D}^{n}\right)$ ?

Let $\mathcal{H}$ be a Hilbert module over $\mathbb{C}[\boldsymbol{z}]$. Denote by $\mathcal{R}(\mathcal{H})$ the set of all non-unitarily equivalent submodules of $\mathcal{H}$, that is, if $\mathcal{S}_{1}, \mathcal{S}_{2} \in \mathcal{R}(\mathcal{H})$ and that $\mathcal{S}_{1} \cong \mathcal{S}_{2}$ then $\mathcal{S}_{1}=\mathcal{S}_{2}$.

\section{Question 3. (Rigidity of submodules) Determine $\mathcal{R}\left(H^{2}\left(\mathbb{D}^{n}\right)\right.$ ).}

The aim of the present paper is to analyze and answer the above questions for the class of co-doubly commuting submodules of $H^{2}\left(\mathbb{D}^{n}\right)$. We obtain an explicit description of the cross commutators of co-doubly commuting submodules of $H^{2}\left(\mathbb{D}^{n}\right)$. As an applications, we prove that the cross commutators of a co-doubly commuting submodule $\mathcal{S}$ of $H^{2}\left(\mathbb{D}^{n}\right)$ are compact, that is, $\mathcal{S}$ is essentially doubly commuting, if and only if $n=2$ or that $\mathcal{S}$ is of finite co-dimension. We would like to point out that a submodule of finite co-dimension is necessarily essentially doubly commuting. Therefore, the issue of essential doubly commutativity of co-doubly commuting submodules of $H^{2}\left(\mathbb{D}^{n}\right)$ yields a rigidity type result: if $\mathcal{S}$ is of infinite co-dimension co-doubly commuting submodules of $H^{2}\left(\mathbb{D}^{n}\right)$ and $\mathcal{S}$ is essentially doubly commuting, then $n=2$ (the base case). Our earlier classification results are also used to prove a Beurling-Lax-Halmos type theorem for the class of co-doubly commuting submodules of $H^{2}\left(\mathbb{D}^{n}\right)$. We also discuss the rigidity phenomenon of such submodules.

Note also that most of the results of the present paper, concerning doubly commuting quotient modules and co-doubly commuting submodules, restricted to the base case $n=2$ are known. However, the proofs are new even in the case $n=2$. Moreover, as we have pointed out above, the difference between the base case $n=2$ and the higher variables case $n>2$ is more curious in the study of essentially doubly commuting submodules of $H^{2}\left(\mathbb{D}^{n}\right)$ (see Corollaries 2.6, 2.7 and 2.9).

We now summarize the contents of this paper. In Section 2 we investigate the essential doubly commutativity problem for the class of co-doubly commuting submodules of $H^{2}\left(\mathbb{D}^{n}\right)$ and conclude that for $n \geq 3$, except for the finite co-dimension case, none of the co-doubly commuting submodules of $H^{2}\left(\mathbb{D}^{n}\right)$ are essentially doubly commuting. In Sections 3 and 4 we answer Questions 2 and 3 for the class of co-doubly commuting submodules of $H^{2}\left(\mathbb{D}^{n}\right)$, respectively. We conclude in Section 5 with some remarks and discussion on the problem of essentially doubly commutativity of Hilbert modules.

\section{Cross commutators of submodules}

In a recent paper [28] we completely classify the class of doubly commuting quotient modules and co-doubly commuting submodules of the Hardy module $H^{2}\left(\mathbb{D}^{n}\right)$, where $n \geq 2$ (see [20] for the case $n=2$ ). 
TheOREM 2.1. Let $\mathcal{Q}$ be a quotient module of $H^{2}\left(\mathbb{D}^{n}\right)$ and $n \geq 2$. Then $\mathcal{Q}$ is doubly commuting quotient module of $H^{2}\left(\mathbb{D}^{n}\right)$ if and only if

$$
\mathcal{Q}=\mathcal{Q}_{\Theta_{1}} \otimes \cdots \otimes \mathcal{Q}_{\Theta_{n}}
$$

where each $\mathcal{Q}_{\Theta_{i}}=H^{2}(\mathbb{D}) / \Theta_{i} H^{2}(\mathbb{D})$, a Jordan block of $H^{2}(\mathbb{D})$ for some inner function $\Theta_{i} \in$ $H^{\infty}(\mathbb{D})$, or $\mathcal{Q}_{\Theta_{i}}=H^{2}(\mathbb{D})$ for all $i=1, \ldots, n$. Moreover, there exists an integer $m \in\{1, \ldots, n\}$ and inner functions $\Theta_{i_{j}} \in H^{\infty}(\mathbb{D})$ such that

$$
\mathcal{Q}^{\perp}=\sum_{1 \leq i_{1}<\ldots<i_{m} \leq n} \tilde{\Theta}_{i_{j}} H^{2}\left(\mathbb{D}^{n}\right)
$$

where $\tilde{\Theta}_{i_{j}}(\boldsymbol{z})=\Theta_{i_{j}}\left(z_{i_{j}}\right)$ for all $\boldsymbol{z} \in \mathbb{D}^{n}$. Finally,

$$
P_{\mathcal{Q}}=I_{H^{2}\left(\mathbb{D}^{n}\right)}-\prod_{j=1}^{m}\left(I_{H^{2}\left(\mathbb{D}^{n}\right)}-M_{\tilde{\Theta}_{i_{j}}} M_{\tilde{\Theta}_{i_{j}}}^{*}\right), \quad \text { and } \quad P_{\mathcal{Q}^{\perp}}=\prod_{j=1}^{m}\left(I_{H^{2}\left(\mathbb{D}^{n}\right)}-M_{\tilde{\Theta}_{i_{j}}} M_{\tilde{\Theta}_{i_{j}}}^{*}\right) .
$$

In what follows, we realize a doubly commuting quotient module $\mathcal{Q}$ of $H^{2}\left(\mathbb{D}^{n}\right)$ as $\mathcal{Q}_{\Theta_{1}} \otimes$ $\cdots \otimes \mathcal{Q}_{\Theta_{n}}$ where each $\mathcal{Q}_{\Theta_{i}}(1 \leq i \leq n)$ is either a Jordan block of $H^{2}(\mathbb{D})$ (see [6], [8], [23]) or the Hardy module $H^{2}(\mathbb{D})$. Consequently, a co-doubly commuting submodule $\mathcal{S}$ of $H^{2}\left(\mathbb{D}^{n}\right)$ will be realized as

$$
\mathcal{S}=\sum_{1 \leq i \leq n} \tilde{\Theta}_{i} H^{2}\left(\mathbb{D}^{n}\right)
$$

where $\tilde{\Theta}_{i}(\boldsymbol{z})=\Theta_{i}\left(z_{i}\right)$ for all $\boldsymbol{z} \in \mathbb{D}^{n}$ and each $\Theta_{i} \in H^{\infty}(\mathbb{D})$ is either inner or the zero function.

Note that a Jordan block $\mathcal{Q}_{\Theta}$ of $H^{2}(\mathbb{D})$ is of finite dimension if and only if the inner function $\Theta$ is a finite Blaschke products on the unit disk. Moreover, for any Jordan block $\mathcal{Q}_{\Theta}$ of $H^{2}(\mathbb{D})$ we have

where $C_{z}=\left.P_{\mathcal{Q}_{\Theta}} M_{z}\right|_{\mathcal{Q}_{\Theta}}$.

$$
\operatorname{rank}\left[C_{z}^{*}, C_{z}\right] \leq 1
$$

First, we record a simple observation concerning essentially normal doubly commuting quotient modules of the Hardy module $H^{2}\left(\mathbb{D}^{n}\right)$.

Proposition 2.2. Let $\mathcal{Q}=\mathcal{Q}_{\Theta_{1}} \otimes \cdots \otimes \mathcal{Q}_{\Theta_{n}}$ be a doubly commuting quotient module of $H^{2}\left(\mathbb{D}^{n}\right)$. Then $\mathcal{Q}$ is essentially normal if and only if each representing function $\Theta_{i}$ of $\mathcal{Q}$ is a finite Blaschke product for all $i=1, \ldots, n$, or, equivalently, $\operatorname{dim} \mathcal{Q}<\infty$.

Proof. Suppose $\mathcal{Q}$ is a doubly commuting quotient module of $H^{2}\left(\mathbb{D}^{n}\right)$, that is,

$$
\left[C_{z_{i}}^{*}, C_{z_{j}}\right]=0
$$

and

$$
C_{z_{i}}=\left.I_{\mathcal{Q}_{\Theta_{1}}} \otimes \cdots \otimes P_{\mathcal{Q}_{\Theta_{i}}} M_{z}\right|_{\mathcal{Q}_{\Theta_{i}}} \otimes \cdots \otimes I_{\mathcal{Q}_{\Theta_{n}}}
$$

for all $1 \leq i<j \leq n$. Then we obtain readily that

$$
\left[C_{z_{i}}^{*}, C_{z_{i}}\right]=I_{\mathcal{Q}_{\Theta_{1}}} \otimes \cdots \otimes \underbrace{\left[C_{z}^{*}, C_{z}\right]}_{i^{t h}} \otimes \cdots \otimes I_{\mathcal{Q}_{\Theta_{n}}},
$$

and conclude that $\left[C_{z_{i}}^{*}, C_{z_{i}}\right] \in \mathcal{K}(\mathcal{Q})$ for all $1 \leq i \leq n$ if and only if $\operatorname{dim} \mathcal{Q}_{\Theta_{i}}<\infty$, or, equivalently, if and only if $\Theta_{i}$ is a finite Blaschke product for all $i=1, \ldots, n$. Hence, $\mathcal{Q}$ is 
essentially normal if and only if $\Theta_{i}$ is a finite Blaschke product for all $i=1, \ldots, n$. This concludes the proof.

Hence it follows in particular that essential normality of submodules of $H^{2}\left(\mathbb{D}^{n}\right)$ seems like a rather strong property. Therefore, in the rest of this section we will focus only on essentially doubly commuting submodules of $H^{2}\left(\mathbb{D}^{n}\right)$.

Before proceeding we need to prove the following result concerning the rank of the multiplication operator restricted to a submodule and projected back onto the corresponding quotient module of the Hardy module $H^{2}(\mathbb{D})$.

Proposition 2.3. Let $\mathcal{Q}_{\Theta}$ be a quotient module of $H^{2}(\mathbb{D})$ for some inner function $\Theta \in$ $H^{\infty}(\mathbb{D})$. Then $C_{\Theta}:=\left.P_{\mathcal{Q}_{\Theta}} M_{z}^{*}\right|_{\Theta H^{2}(\mathbb{D})} \in \mathcal{L}\left(\Theta H^{2}(\mathbb{D}), \mathcal{Q}_{\Theta}\right)$ is given by

$$
C_{\Theta}=\left[M_{z}^{*}, M_{\Theta}\right] M_{\Theta}^{*} \text {. }
$$

Moreover, $C_{\Theta}$ is a rank one operator and

$$
\left\|C_{\Theta}\right\|=\left(1-|\Theta(0)|^{2}\right)^{\frac{1}{2}} .
$$

Proof. We begin by calculating

$$
\begin{aligned}
\left(I-M_{\Theta} M_{\Theta}^{*}\right) M_{z}^{*} M_{\Theta} & =M_{z}^{*} M_{\Theta}-M_{\Theta} M_{\Theta}^{*} M_{z}^{*} M_{\Theta} \\
& =M_{z}^{*} M_{\Theta}-M_{\Theta} M_{z}^{*} M_{\Theta} M_{\Theta}^{*} \\
& =M_{z}^{*} M_{\Theta}-M_{\Theta} M_{z}^{*} \\
& =\left[\left(M_{z}^{*} M_{\Theta}-M_{\Theta} M_{z}^{*}\right) M_{\Theta}^{*}\right] M_{\Theta}
\end{aligned}
$$

Therefore, we have

$$
C_{\Theta}=\left(M_{z}^{*} M_{\Theta}-M_{\Theta} M_{z}^{*}\right) M_{\Theta}^{*}=\left[M_{z}^{*}, M_{\Theta}\right] M_{\Theta}^{*} .
$$

Now for all $l \geq 1$

$$
\left[M_{z}^{*}, M_{\Theta}\right] z^{l}=\left(M_{z}^{*} M_{\Theta}-M_{\Theta} M_{z}^{*}\right) M_{z}^{l} 1=0
$$

and

And so,

$$
\left[M_{z}^{*}, M_{\Theta}\right] 1=\left(M_{z}^{*} M_{\Theta}-M_{\Theta} M_{z}^{*}\right) 1=M_{z}^{*} \Theta
$$

$$
\left[M_{z}^{*}, M_{\Theta}\right] f=M_{z}^{*} M_{\Theta} f(0)=f(0) M_{z}^{*} \Theta=\langle f, 1\rangle M_{z}^{*} \Theta=\langle\Theta f, \Theta\rangle M_{z}^{*} \Theta,
$$

for all $f \in H^{2}(\mathbb{D})$. Hence, we infer that

$$
C_{\Theta}(\Theta f)=\left[M_{z}^{*}, M_{\Theta}\right] f=\langle\Theta f, \Theta\rangle M_{z}^{*} \Theta .
$$

Therefore, $C_{\Theta}$ is a rank one operator and

$$
C_{\Theta} f=\langle f, \Theta\rangle M_{z}^{*} \Theta
$$

for all $f \in \Theta H^{2}(\mathbb{D})$. Finally,

$$
\begin{aligned}
\left\|C_{\Theta}\right\|^{2} & =\|\Theta\|^{2}\left\|M_{z}^{*} \Theta\right\|^{2}=\left\|M_{z}^{*} \Theta\right\|^{2} \\
& =\left\langle M_{z} M_{z}^{*} \Theta, \Theta\right\rangle=\left\langle\left(I_{H^{2}(\mathbb{D})}-P_{\mathbb{C}}\right) \Theta, \Theta\right\rangle \\
& =\|\Theta\|^{2}-|\Theta(0)|^{2} \\
& =1-|\Theta(0)|^{2} .
\end{aligned}
$$


This completes the proof.

In the sequel we will need the following well known fact (cf. Lemma 2.5 in [28]).

Lemma 2.4. Let $\left\{P_{i}\right\}_{i=1}^{n}$ be a collection of commuting orthogonal projections on a Hilbert space $\mathcal{H}$. Then

$$
\mathcal{L}:=\sum_{i=1}^{n} \operatorname{ran} P_{i}
$$

is closed and the orthogonal projection of $\mathcal{H}$ onto $\mathcal{L}$ is given by

$$
\begin{aligned}
P_{\mathcal{L}} & =P_{1}\left(I-P_{2}\right) \cdots\left(I-P_{n}\right)+P_{2}\left(I-P_{3}\right) \cdots\left(I-P_{n}\right)+\cdots+P_{n-1}\left(I-P_{n}\right)+P_{n} \\
& =P_{n}\left(I-P_{n-1}\right) \cdots\left(I-P_{1}\right)+P_{n-1}\left(I-P_{n-2}\right) \cdots\left(I-P_{1}\right)+\cdots+P_{2}\left(I-P_{1}\right)+P_{1} .
\end{aligned}
$$

Moreover,

$$
P_{\mathcal{L}}=I-\prod_{i=1}^{n}\left(I-P_{i}\right)
$$

We now are ready to compute the cross commutators of a co-doubly commuting submodule of $H^{2}\left(\mathbb{D}^{n}\right)$.

ThEOREM 2.5. Let $\mathcal{S}=\sum_{i=1}^{n} \tilde{\Theta}_{i} H^{2}\left(\mathbb{D}^{n}\right)$ be a co-doubly commuting submodule of $H^{2}\left(\mathbb{D}^{n}\right)$, where $\tilde{\Theta}_{i}(\boldsymbol{z})=\Theta_{i}\left(z_{i}\right)$ for all $\boldsymbol{z} \in \mathbb{D}^{n}$ and each $\Theta_{i} \in H^{\infty}(\mathbb{D})$ is either an inner function or the zero function and $1 \leq i \leq n$. Then for all $1 \leq i<j \leq n$,

$$
\left[R_{z_{i}}^{*}, R_{z_{j}}\right]=I_{\mathcal{Q}_{\Theta_{1}}} \otimes \cdots \otimes \underbrace{\left.P_{\mathcal{Q}_{\Theta_{i}}} M_{z}^{*}\right|_{\Theta_{i} H^{2}(\mathbb{D})}}_{i^{\text {th }}} \otimes \cdots \otimes \underbrace{\left.P_{\Theta_{j} H^{2}(\mathbb{D})} M_{z}\right|_{\mathcal{Q}_{\Theta_{j}}}}_{j^{\text {th }}} \otimes \cdots \otimes I_{\mathcal{Q}_{\Theta_{n}}},
$$

and

$$
\left\|\left[R_{z_{i}}^{*}, R_{z_{j}}\right]\right\|=\left(1-\left|\Theta_{i}(0)\right|^{2}\right)^{\frac{1}{2}}\left(1-\left|\Theta_{j}(0)\right|^{2}\right)^{\frac{1}{2}} .
$$

Proof. Let $\mathcal{S}=\sum_{i=1}^{n} \tilde{\Theta}_{i} H^{2}\left(\mathbb{D}^{n}\right)$, for some one variable inner functions $\Theta_{i} \in H^{\infty}(\mathbb{D})$. Let $\tilde{P}_{i}$ be the orthogonal projection in $\mathcal{L}(\mathcal{S})$ defined by

$$
\tilde{P}_{i}=M_{\tilde{\Theta}_{i}} M_{\tilde{\Theta}_{i}}^{*},
$$

for all $i=1, \ldots, n$. Then it follows that $\left\{\tilde{P}_{i}\right\}_{i=1}^{n}$ is a collection of commuting orthogonal projections. By virtue of Theorem 2.1 and Lemma 2.4,

$$
\begin{aligned}
P_{\mathcal{S}} & =I_{H^{2}\left(\mathbb{D}^{n}\right)}-\prod_{i=1}^{n}\left(I_{H^{2}\left(\mathbb{D}^{n}\right)}-\tilde{P}_{i}\right) \\
& =\tilde{P}_{1}\left(I-\tilde{P}_{2}\right) \cdots\left(I-\tilde{P}_{n}\right)+\tilde{P}_{2}\left(I-\tilde{P}_{3}\right) \cdots\left(I-\tilde{P}_{n}\right)+\cdots+\tilde{P}_{n-1}\left(I-\tilde{P}_{n}\right)+\tilde{P}_{n} \\
& =\tilde{P}_{n}\left(I-\tilde{P}_{n-1}\right) \cdots\left(I-\tilde{P}_{1}\right)+\tilde{P}_{n-1}\left(I-\tilde{P}_{n-2}\right) \cdots\left(I-\tilde{P}_{1}\right)+\cdots+\tilde{P}_{2}\left(I-\tilde{P}_{1}\right)+\tilde{P}_{1},
\end{aligned}
$$

and

$$
P_{\mathcal{Q}}=\prod_{i=1}^{n}\left(I_{H^{2}\left(\mathbb{D}^{n}\right)}-\tilde{P}_{i}\right) .
$$

On the other hand, for all $1 \leq i<j \leq n$, we obtain

$$
\left[R_{z_{i}}^{*}, R_{z_{j}}\right]=\left.P_{\mathcal{S}} M_{z_{i}}^{*} M_{z_{j}}\right|_{\mathcal{S}}-\left.P_{\mathcal{S}} M_{z_{j}} P_{\mathcal{S}} M_{z_{i}}^{*}\right|_{\mathcal{S}}
$$


and that

$$
\begin{aligned}
P_{\mathcal{S}} M_{z_{i}}^{*} M_{z_{j}} P_{\mathcal{S}}-P_{\mathcal{S}} M_{z_{j}} P_{\mathcal{S}} M_{z_{i}}^{*} P_{\mathcal{S}} & =P_{\mathcal{S}} M_{z_{i}}^{*} M_{z_{j}} P_{\mathcal{S}}-P_{\mathcal{S}} M_{z_{j}}\left(I-P_{\mathcal{Q}}\right) M_{z_{i}}^{*} P_{\mathcal{S}} \\
& =P_{\mathcal{S}} M_{z_{j}} P_{\mathcal{Q}} M_{z_{i}}^{*} P_{\mathcal{S}} .
\end{aligned}
$$

Furthermore we have for all $1 \leq i<j \leq n$,

$$
\begin{aligned}
& P_{\mathcal{S}} M_{z_{j}} P_{\mathcal{Q}} M_{z_{i}}^{*} P_{\mathcal{S}} \\
&=\left[\tilde{P}_{n}\left(I-\tilde{P}_{n-1}\right) \cdots\left(I-\tilde{P}_{1}\right)+\tilde{P}_{n-1}\left(I-\tilde{P}_{n-2}\right) \cdots\left(I-\tilde{P}_{1}\right)+\cdots+\tilde{P}_{2}\left(I-\tilde{P}_{1}\right)+\tilde{P}_{1}\right] \\
& M_{z_{j}}\left[\prod_{l=1}^{n}\left(I_{H^{2}\left(\mathbb{D}^{n}\right)}-\tilde{P}_{l}\right)\right] M_{z_{i}}^{*} \\
& {\left[\tilde{P}_{1}\left(I-\tilde{P}_{2}\right) \cdots\left(I-\tilde{P}_{n}\right)+\tilde{P}_{2}\left(I-\tilde{P}_{3}\right) \cdots\left(I-\tilde{P}_{n}\right)+\cdots+\tilde{P}_{n-1}\left(I-\tilde{P}_{n}\right)+\tilde{P}_{n}\right] } \\
&= {\left[\tilde{P}_{n}\left(I-\tilde{P}_{n-1}\right) \cdots\left(I-\tilde{P}_{1}\right)+\tilde{P}_{n-1}\left(I-\tilde{P}_{n-2}\right) \cdots\left(I-\tilde{P}_{1}\right)+\cdots+\tilde{P}_{2}\left(I-\tilde{P}_{1}\right)+\tilde{P}_{1}\right] } \\
& {\left[\prod_{l \neq j}\left(I_{H^{2}}\left(\mathbb{D}^{n}\right)-\tilde{P}_{l}\right)\right] M_{z_{j}} M_{z_{i}}^{*}\left[\prod_{l \neq i}\left(I_{H^{2}\left(\mathbb{D}^{n}\right)}-\tilde{P}_{l}\right)\right] } \\
& {\left[\tilde{P}_{1}\left(I-\tilde{P}_{2}\right) \cdots\left(I-\tilde{P}_{n}\right)+\tilde{P}_{2}\left(I-\tilde{P}_{3}\right) \cdots\left(I-\tilde{P}_{n}\right)+\cdots+\tilde{P}_{n-1}\left(I-\tilde{P}_{n}\right)+\tilde{P}_{n}\right] } \\
&= {\left[\tilde{P}_{j}\left(I-\tilde{P}_{j-1}\right) \cdots\left(I-\tilde{P}_{1}\right)\right] M_{z_{i}}^{*} M_{z_{j}}\left(\tilde{P}_{i}\left(I-\tilde{P}_{i+1}\right) \cdots\left(I-\tilde{P}_{n}\right)\right] } \\
&= {\left[\left(I-\tilde{P}_{1}\right) \cdots\left(I-\tilde{P}_{j-1}\right) \tilde{P}_{j}\right] M_{z_{i}}^{*} M_{z_{j}}\left[\tilde{P}_{i}\left(I-\tilde{P}_{i+1}\right) \cdots\left(I-\tilde{P}_{n}\right)\right] . }
\end{aligned}
$$

These equalities shows that

$$
\begin{aligned}
{\left[R_{z_{i}}^{*}, R_{z_{j}}\right]=} & {\left[\left(I-\tilde{P}_{1}\right) \cdots\left(I-\tilde{P}_{i}\right) \cdots\left(I-\tilde{P}_{j-1}\right) \tilde{P}_{j}\right] M_{z_{i}}^{*} M_{z_{j}}\left[\tilde{P}_{i}\left(I-\tilde{P}_{i+1}\right) \cdots\left(I-\tilde{P}_{j}\right) \cdots\left(I-\tilde{P}_{n}\right)\right] } \\
= & \left(I-\tilde{P}_{1}\right)\left(I-\tilde{P}_{2}\right) \cdots\left(I-\tilde{P}_{i-1}\right)\left(\left(I-\tilde{P}_{i}\right) M_{z_{i}}^{*} \tilde{P}_{i}\right)\left(I-\tilde{P}_{i+1}\right) \cdots \\
& \cdots\left(I-\tilde{P}_{j-1}\right)\left(\tilde{P}_{j} M_{z_{j}}\left(I-\tilde{P}_{j}\right)\right)\left(I-\tilde{P}_{j+1}\right) \cdots\left(I-\tilde{P}_{n}\right) .
\end{aligned}
$$

Moreover,

$$
\left[R_{z_{i}}^{*}, R_{z_{j}}\right]=\left[\left(I-\tilde{P}_{1}\right) \cdots\left(I-\tilde{P}_{j-1}\right) \tilde{P}_{j}\right] M_{z_{i}}^{*} M_{z_{j}}\left[\left(I-\tilde{P}_{1}\right) \cdots\left(I-\tilde{P}_{i-1}\right) \tilde{P}_{i}\left(I-\tilde{P}_{i+1}\right) \cdots\left(I-\tilde{P}_{n}\right)\right],
$$

and

$$
\left[R_{z_{i}}^{*}, R_{z_{j}}\right]=\left[\left(I-\tilde{P}_{1}\right) \cdots\left(I-\tilde{P}_{j-1}\right) \tilde{P}_{j}\left(I-\tilde{P}_{j+1}\right) \cdots\left(I-\tilde{P}_{n}\right)\right] M_{z_{i}}^{*} M_{z_{j}}\left[\tilde{P}_{i}\left(I-\tilde{P}_{i+1}\right) \cdots\left(I-\tilde{P}_{n}\right)\right] .
$$

We conclude that the cross-commutator $\left[R_{z_{i}}^{*}, R_{z_{j}}\right]$ is a bounded linear operator from

$$
\mathcal{Q}_{\Theta_{1}} \otimes \cdots \otimes \mathcal{Q}_{\Theta_{i-1}} \otimes \Theta_{i} H^{2}(\mathbb{D}) \otimes \mathcal{Q}_{\Theta_{i+1}} \otimes \cdots \otimes \mathcal{Q}_{\Theta_{j}} \otimes \cdots \otimes \mathcal{Q}_{\Theta_{n}} \subseteq \mathcal{S}
$$

to

$$
\mathcal{Q}_{\Theta_{1}} \otimes \cdots \otimes \mathcal{Q}_{\Theta_{i}} \otimes \cdots \otimes \mathcal{Q}_{\Theta_{j-1}} \otimes \Theta_{j} H^{2}(\mathbb{D}) \otimes \mathcal{Q}_{\Theta_{j+1}} \otimes \cdots \otimes \mathcal{Q}_{\Theta_{n}} \subseteq \mathcal{S}
$$

and

$$
\left[R_{z_{i}}^{*}, R_{z_{j}}\right]=I_{\mathcal{Q}_{\Theta_{1}}} \otimes \cdots \otimes \underbrace{\left.P_{\mathcal{Q}_{\Theta_{i}}} M_{z}^{*}\right|_{\Theta_{i} H^{2}(\mathbb{D})}}_{i^{\text {th }}} \otimes \cdots \otimes \underbrace{\left.P_{\Theta_{j} H^{2}(\mathbb{D})} M_{z}\right|_{\mathcal{Q}_{\Theta_{j}}}}_{j^{\text {th }}} \otimes \cdots \otimes I_{\mathcal{Q}_{\Theta_{n}}} .
$$

Further, we note that

$$
\begin{aligned}
\left\|\left[R_{z_{i}}^{*}, R_{z_{j}}\right]\right\| & =\left\|\left.\left.I_{\mathcal{Q}_{\Theta_{1}}} \otimes \cdots \otimes P_{\mathcal{Q}_{\Theta_{i}}} M_{z}^{*}\right|_{\Theta_{i} H^{2}(\mathbb{D})} \otimes \cdots \otimes P_{\Theta_{j} H^{2}(\mathbb{D})} M_{z}\right|_{\mathcal{Q}_{\Theta_{j}}} \otimes \cdots \otimes I_{\mathcal{Q}_{\Theta_{n}}}\right\| \\
& =\left\|\left.P_{\mathcal{Q}_{\Theta_{i}}} M_{z}^{*}\right|_{\Theta_{i} H^{2}(\mathbb{D})}\right\|\left\|\left.P_{\Theta_{j} H^{2}(\mathbb{D})} M_{z}\right|_{\mathcal{Q}_{j}}\right\|,
\end{aligned}
$$


and consequently by Proposition 2.3 we have

$$
\left\|\left[R_{z_{i}}^{*}, R_{z_{j}}\right]\right\|=\left(1-\left|\Theta_{i}(0)\right|^{2}\right)^{\frac{1}{2}}\left(1-\left|\Theta_{j}(0)\right|^{2}\right)^{\frac{1}{2}} .
$$

This completes the proof.

In the following corollary we reveal the significance of the identity operators in the cross commutators of the co-doubly commuting submodules of $H^{2}\left(\mathbb{D}^{n}\right)$ for $n \geq 2$.

Corollary 2.6. Let $\mathcal{S}=\sum_{i=1}^{n} \tilde{\Theta}_{i} H^{2}\left(\mathbb{D}^{n}\right)$ be a submodule of $H^{2}\left(\mathbb{D}^{n}\right)$ for some one variable inner functions $\left\{\tilde{\Theta}_{i}\right\}_{i=1}^{n} \subseteq H^{\infty}\left(\mathbb{D}^{n}\right)$. Then

(1) for $n=2$ : the rank of the cross commutator of $\mathcal{S}$ is at most one and the Hilbert-Schmidt norm of the cross commutator is given by

$$
\left\|\left[R_{z_{1}}^{*}, R_{z_{2}}\right]\right\|_{H S}=\left(1-\left|\Theta_{1}(0)\right|^{2}\right)^{\frac{1}{2}}\left(1-\left|\Theta_{2}(0)\right|^{2}\right)^{\frac{1}{2}} .
$$

In particular, $\mathcal{S}$ is essentially doubly commuting.

(2) for $n>2: \mathcal{S}$ is essentially doubly commuting (or of Hilbert-Schmidt cross-commutators) if and only if $\tilde{\Theta}_{i}$ is a one variable finite Blaschke product for all $1 \leq i \leq n$, if and only if that $\mathcal{S}$ is of finite co-dimension, that is,

$$
\operatorname{dim}\left[H^{2}\left(\mathbb{D}^{n}\right) / \mathcal{S}\right]<\infty .
$$

Moreover, in this case, for all $1 \leq i<j \leq n$

$$
\left\|\left[R_{z_{i}}^{*}, R_{z_{j}}\right]\right\|_{H S}=\left(1-\left|\Theta_{i}(0)\right|^{2}\right)^{\frac{1}{2}}\left(1-\left|\Theta_{j}(0)\right|^{2}\right)^{\frac{1}{2}} .
$$

Part (1) of the above corollary was obtained by R. Yang (Corollary 1.1, [30]). We refer the reader to [1] for more details on finite co-dimensional submodules of the Hardy modules over $\mathbb{D}^{n}$.

As another consequence of the above theorem, we have the following.

Corollary 2.7. Let $n>2$ and $\mathcal{S}=\sum_{i=1}^{k} \tilde{\Theta}_{i} H^{2}\left(\mathbb{D}^{n}\right)$ be a co-doubly commuting proper submodule of $H^{2}\left(\mathbb{D}^{n}\right)$ for some inner functions $\left\{\Theta_{i}\right\}_{i=1}^{k}$ and $k<n$. Then $\mathcal{S}$ is not essentially doubly commuting.

Combining Corollary 2.6 and Proposition 2.2 we obtain:

Corollary 2.8. Let $\mathcal{S}$ be a co-doubly commuting submodule of $H^{2}\left(\mathbb{D}^{n}\right)$ and $\mathcal{Q}:=H^{2}\left(\mathbb{D}^{n}\right) / \mathcal{S}$ and $n>2$. Then the following are equivalent:

(i) $\mathcal{S}$ is essentially doubly commuting.

(ii) $\mathcal{S}$ is of finite co-dimension.

(iii) $\mathcal{Q}$ is essentially normal.

We conclude this section with a "rigidity" result.

Corollary 2.9. Let $n \geq 2$ and $\mathcal{S}=\sum_{i=1}^{n} \tilde{\Theta}_{i} H^{2}\left(\mathbb{D}^{n}\right)$ be an essentially normal co-doubly commuting submodule of $H^{2}\left(\mathbb{D}^{n}\right)$ for some one variable inner functions $\left\{\Theta_{i}\right\}_{i=1}^{n}$. If $\mathcal{S}$ is of infinite co-dimensional, then $n=2$.

Proof. The result follows from the implication (i) $\Longrightarrow$ (ii) of Corollary 2.8. 


\section{Representing Inner functions of Submodules}

In this section, we will obtain the explicit representations of the Beurling-Lax-Halmos inner functions of a class of submodules of $H_{\mathcal{E}}^{2}(\mathbb{D})$. if

Recall that a non-trivial closed subspace $\mathcal{S}$ of $H_{\mathcal{E}}^{2}(\mathbb{D})$ is a submodule of $H_{\mathcal{E}}^{2}(\mathbb{D})$ if and only

$$
\mathcal{S}=\Theta H_{\mathcal{E}_{*}}^{2}(\mathbb{D})
$$

for some closed subspace $\mathcal{E}_{*}$ of $\mathcal{E}$ and inner function $\Theta \in H_{\mathcal{L}\left(\mathcal{E}_{*}, \mathcal{E}\right)}^{\infty}(\mathbb{D})$ (unique up to unitary equivalence). This fact is known as the Beurling-Lax-Halmos theorem and that $\Theta$ as the representing inner function of the submodule $\mathcal{S}$. Given a submodule $\mathcal{S}$ of $H_{\mathcal{E}}^{2}(\mathbb{D})$, it is a question of interest to determine the inner function $\Theta$ associated with $\mathcal{S}$.

Now let $\mathcal{S}$ be a co-doubly commuting submodule of $H^{2}\left(\mathbb{D}^{n}\right)$. Then by Theorem 2.1 we have

$$
\mathcal{S}=\sum_{i=1}^{n} \tilde{\Theta}_{i} H^{2}\left(\mathbb{D}^{n}\right),
$$

where $\tilde{\Theta}_{i} \in H^{\infty}\left(\mathbb{D}^{n}\right)$ is either the zero function or one variable inner function and $i=$ $1, \ldots, n$. We realize $\mathcal{S}$ as a submodule of $H_{\mathcal{E}}^{2}(\mathbb{D})$ where $\mathcal{E}=H^{2}\left(\mathbb{D}^{n-1}\right)$. Then by the BeurlingLax-Halmos theorem, there exists an inner function $\Theta \in H_{\mathcal{L}\left(\mathcal{E}_{*}, H^{2}\left(\mathbb{D}^{n-1}\right)\right)}^{\infty}(\mathbb{D})$, for some closed subspace $\mathcal{E}_{*}$ of $H^{2}\left(\mathbb{D}^{n-1}\right)$ such that

$$
\mathcal{S}=\sum_{i=1}^{n} \tilde{\Theta}_{i} H^{2}\left(\mathbb{D}^{n}\right)=\Theta H_{\mathcal{E}_{*}}^{2}(\mathbb{D}) .
$$

Since

$$
R_{z} R_{z}^{*}=\left.M_{z} P_{\mathcal{S}} M_{z}^{*}\right|_{\mathcal{S}}=M_{z} P_{\mathcal{S}} M_{z}^{*}
$$

that $R_{z} R_{z}^{*}$ is an orthogonal projection onto $z \mathcal{S}$ and hence we have the orthogonal projection

$$
P_{\mathcal{S}}-R_{z} R_{z}^{*}=P_{\mathcal{S} \ominus z \mathcal{S}}
$$

On the other hand

$$
\begin{aligned}
P_{\mathcal{S}}-R_{z} R_{z}^{*} & =M_{\Theta} M_{\Theta}^{*}-M_{z} M_{\Theta} M_{\Theta}^{*} M_{z}^{*}=M_{\Theta}\left(I_{H_{\mathcal{E}_{*}}^{2}(\mathbb{D})}-M_{z} M_{z}^{*}\right) M_{\Theta}^{*}=M_{\Theta} P_{\mathcal{E}_{*}} M_{\Theta}^{*} \\
& =\left(M_{\Theta} P_{\mathcal{E}_{*}}\right)\left(M_{\Theta} P_{\mathcal{E}_{*}}\right)^{*},
\end{aligned}
$$

and hence

$$
\begin{aligned}
\mathcal{S} \ominus z \mathcal{S} & =\operatorname{ran}\left(P_{\mathcal{S}}-R_{z} R_{z}^{*}\right)=\operatorname{ran}\left(M_{\Theta} P_{\mathcal{E}_{*}} M_{\Theta}^{*}\right)=\operatorname{ran}\left(M_{\Theta} P_{\mathcal{E}_{*}}\right) \\
& =\left\{\Theta \eta: \eta \in \mathcal{E}_{*}\right\} .
\end{aligned}
$$

Note also that $\mathcal{S} \ominus z \mathcal{S}$ is the wandering subspace of $\mathcal{S}$, that is,

$$
\mathcal{S}=\overline{\operatorname{span}}\left\{z^{l}(\mathcal{S} \ominus z \mathcal{S}): l \geq 0\right\}=\Theta H_{\mathcal{E}_{*}}^{2}(\mathbb{D}) .
$$

After these preliminaries we can turn to the proof of the main result of this section.

THEOREM 3.1. Let $\mathcal{S}$ be a submodule of $H_{H^{2}\left(\mathbb{D}^{n-1}\right)}^{2}(\mathbb{D})$ with the Beurling-Lax-Halmos representation $\mathcal{S}=\Theta H_{\mathcal{E}}^{2}(\mathbb{D})$ for some closed subspace $\mathcal{E}$ of $H^{2}\left(\mathbb{D}^{n-1}\right)$ and inner function $\Theta \in$ $H_{\mathcal{B}\left(\mathcal{E}, H^{2}\left(\mathbb{D}^{n-1}\right)\right)}^{\infty}(\mathbb{D})$. Then $\mathcal{S} \subseteq H^{2}\left(\mathbb{D}^{n}\right)$ is a co-doubly commuting submodule of $H^{2}\left(\mathbb{D}^{n}\right)$ if and 
only if there exits an integer $m \leq n$ and orthogonal projections $\left\{P_{2}, \ldots, P_{m}\right\}$ in $\mathcal{L}\left(H^{2}(\mathbb{D})\right)$ and an inner function $\Theta_{1} \in H^{\infty}(\mathbb{D})$ such that $\mathcal{E}=H^{2}\left(\mathbb{D}^{n-1}\right)$ and

$$
\Theta(z)=\Theta_{1}(z)\left(I-\tilde{P}_{2}\right) \cdots\left(I-\tilde{P}_{m}\right)+\tilde{P}_{2}\left(I-\tilde{P}_{3}\right) \cdots\left(I-\tilde{P}_{m}\right)+\cdots+\tilde{P}_{m-1}\left(I-\tilde{P}_{m}\right)+\tilde{P}_{m},
$$

for all $z \in \mathbb{D}$, where

$$
\tilde{P}_{i}=I_{H^{2}(\mathbb{D})} \otimes \cdots \otimes \underset{(i-1)^{t h}}{P_{i}} \otimes \cdots \otimes I_{H^{2}(\mathbb{D})} \in \mathcal{L}\left(H^{2}\left(\mathbb{D}^{n-1}\right) .\right.
$$

Proof. Let $\mathcal{S}$ be a co-doubly commuting submodule of $H^{2}\left(\mathbb{D}^{n}\right)$ so that

$$
\mathcal{S}=\sum_{1 \leq i_{1}<\cdots<i_{m} \leq n} \tilde{\Theta}_{i_{j}} H^{2}\left(\mathbb{D}^{n}\right),
$$

for some one variable inner function $\tilde{\Theta}_{i_{j}} \in H^{\infty}\left(\mathbb{D}^{n}\right)$ and $1 \leq i_{1}<\cdots<i_{m} \leq n$. Without loss of generality, we assume that $i_{j}=j$ for all $j=1, \ldots, m$, that is,

$$
\mathcal{S}=\sum_{j=1}^{m} \tilde{\Theta}_{j} H^{2}\left(\mathbb{D}^{n}\right) .
$$

Then Theorem 2.1 implies that

$$
\begin{aligned}
P_{\mathcal{S}} & =I_{H^{2}\left(\mathbb{D}^{n}\right)}-\prod_{j=1}^{m}\left(I_{H^{2}\left(\mathbb{D}^{n}\right)}-M_{\tilde{\Theta}_{j}} M_{\tilde{\Theta}_{j}}^{*}\right) \\
& =I_{H^{2}\left(\mathbb{D}^{n}\right)}-\left(I_{H^{2}\left(\mathbb{D}^{n}\right)}-M_{\tilde{\Theta}_{1}} M_{\tilde{\Theta}_{1}}^{*}\right) \prod_{j=2}^{m}\left(I_{H^{2}\left(\mathbb{D}^{n}\right)}-I_{H^{2}(\mathbb{D})} \otimes \tilde{P}_{j}\right),
\end{aligned}
$$

where

$$
\tilde{P}_{j}=\underbrace{I_{H^{2}(\mathbb{D})} \otimes \cdots \otimes M_{\Theta_{j}} M_{\Theta_{j}}^{*} \otimes \cdots I_{H^{2}(\mathbb{D})}}_{(n-1) \text { times }} \in \mathcal{L}\left(H^{2}\left(\mathbb{D}^{n-1}\right)\right),
$$

for all $j=2, \ldots, m$. Define $\Theta \in H_{\mathcal{L}\left(H^{2}\left(\mathbb{D}^{n-1}\right)\right)}^{\infty}(\mathbb{D})$ by

$$
\Theta(z)=\Theta_{1}(z)\left(I-\tilde{P}_{2}\right) \cdots\left(I-\tilde{P}_{m}\right)+\tilde{P}_{2}\left(I-\tilde{P}_{3}\right) \cdots\left(I-\tilde{P}_{m}\right)+\cdots+\tilde{P}_{m-1}\left(I-\tilde{P}_{m}\right)+\tilde{P}_{m},
$$

for all $z \in \mathbb{D}$. First, note that

$$
M_{\Theta}=M_{\Theta_{1}}\left(I-\tilde{P}_{2}\right) \cdots\left(I-\tilde{P}_{m}\right)+\tilde{P}_{2}\left(I-\tilde{P}_{3}\right) \cdots\left(I-\tilde{P}_{m}\right)+\cdots+\tilde{P}_{m-1}\left(I-\tilde{P}_{m}\right)+\tilde{P}_{m} .
$$

Since the terms in the sum are orthogonal projection with orthogonal ranges, we compute

$$
\begin{aligned}
M_{\Theta}^{*} M_{\Theta} & =M_{\Theta_{1}}^{*} M_{\Theta_{1}}\left(I-\tilde{P}_{2}\right) \cdots\left(I-\tilde{P}_{m}\right)+\tilde{P}_{2}\left(I-\tilde{P}_{3}\right) \cdots\left(I-\tilde{P}_{m}\right)+\cdots+\tilde{P}_{m-1}\left(I-\tilde{P}_{m}\right)+\tilde{P}_{m} \\
& =\left(I-\tilde{P}_{2}\right) \cdots\left(I-\tilde{P}_{m}\right)+\tilde{P}_{2}\left(I-\tilde{P}_{3}\right) \cdots\left(I-\tilde{P}_{m}\right)+\cdots+\tilde{P}_{m-1}\left(I-\tilde{P}_{m}\right)+\tilde{P}_{m} \\
& =\prod_{j=2}^{m}\left(I_{H^{2}\left(\mathbb{D}^{n}\right)}-\tilde{P}_{j}\right)+\left(I_{H^{2}\left(\mathbb{D}^{n}\right)}-\prod_{j=2}^{m}\left(I_{H^{2}\left(\mathbb{D}^{n}\right)}-\tilde{P}_{j}\right)\right. \\
& =I_{H^{2}\left(\mathbb{D}^{n-1}\right)}
\end{aligned}
$$


and hence that $\Theta$ is an inner function. To prove that $\Theta$ is the Beurling-Lax-Halmos representing inner function of $\mathcal{S}$, by virtue of (3.1), it is enough to show that

$$
\overline{\operatorname{span}}\left\{z^{l} \Theta H^{2}\left(\mathbb{D}^{n-1}\right): l \geq 0\right\}=\sum_{j=1}^{m} \tilde{\Theta}_{j} H^{2}\left(\mathbb{D}^{n}\right) .
$$

Observe that

$$
\begin{aligned}
\Theta H^{2}\left(\mathbb{D}^{n-1}\right)= & \Theta_{1}(\mathcal{Q}_{\Theta_{2}} \otimes \cdots \otimes \mathcal{Q}_{\Theta_{m}} \otimes \underbrace{H^{2}(\mathbb{D}) \otimes \cdots \otimes H^{2}(\mathbb{D})}_{(n-m) \text { times }}) \\
& \oplus(\Theta_{2} H^{2}(\mathbb{D}) \otimes \mathcal{Q}_{\Theta_{3}} \otimes \cdots \otimes \mathcal{Q}_{\Theta_{m}} \otimes \underbrace{H^{2}(\mathbb{D}) \otimes \cdots \otimes H^{2}(\mathbb{D})}_{(n-m) \text { times }}) \\
& \oplus \cdots \oplus(H^{2}(\mathbb{D}) \otimes \cdots \otimes H^{2}(\mathbb{D}) \otimes \Theta_{m} H^{2}(\mathbb{D}) \otimes \underbrace{H^{2}(\mathbb{D}) \otimes \cdots \otimes H^{2}(\mathbb{D})}_{(n-m) \text { times }}),
\end{aligned}
$$

and hence

$$
\begin{aligned}
\overline{\operatorname{span}}\left\{z^{l} \Theta\right. & \left.H^{2}\left(\mathbb{D}^{n-1}\right): l \geq 0\right\}=(\Theta_{1} H^{2}(\mathbb{D}) \otimes \mathcal{Q}_{\Theta_{2}} \otimes \cdots \otimes \mathcal{Q}_{\Theta_{m}} \otimes \underbrace{H^{2}(\mathbb{D}) \otimes \cdots \otimes H^{2}(\mathbb{D})}_{(n-m) \text { times }}) \\
& \oplus(H^{2}(\mathbb{D}) \otimes \Theta_{2} H^{2}(\mathbb{D}) \otimes \mathcal{Q}_{\Theta_{3}} \otimes \cdots \otimes \mathcal{Q}_{\Theta_{m}} \otimes \underbrace{H^{2}(\mathbb{D}) \otimes \cdots \otimes H^{2}(\mathbb{D})}_{(n-m) \text { times }}) \oplus \\
& \cdots \oplus(H^{2}(\mathbb{D}) \otimes \cdots \otimes H^{2}(\mathbb{D}) \otimes \Theta_{m} H^{2}(\mathbb{D}) \otimes \underbrace{H^{2}(\mathbb{D}) \otimes \cdots \otimes H^{2}(\mathbb{D})}_{(n-m) \text { times }}) \\
= & \operatorname{ran}\left[I_{H^{2}\left(\mathbb{D}^{n}\right)}-\prod_{j=1}^{m}\left(I_{H^{2}\left(\mathbb{D}^{n}\right)}-M_{\tilde{\Theta}_{j}} M_{\tilde{\Theta}_{j}}^{*}\right)\right] \\
= & \sum_{i=1}^{m} \tilde{\Theta}_{i} H^{2}\left(\mathbb{D}^{n}\right) .
\end{aligned}
$$

Conversely, if $\Theta$ is given as above, then we realize $\Theta \in H_{\mathcal{L}\left(H^{2}\left(\mathbb{D}^{n-1}\right)\right)}^{\infty}(\mathbb{D})$ by $\tilde{\Theta} \in H^{\infty}\left(\mathbb{D}^{n}\right)$ where

$$
\tilde{\Theta}(\boldsymbol{z})=\Theta_{1}\left(z_{1}\right)\left(I-\tilde{P}_{2}\right) \cdots\left(I-\tilde{P}_{m}\right)+\tilde{P}_{2}\left(I-\tilde{P}_{3}\right) \cdots\left(I-\tilde{P}_{m}\right)+\cdots+\tilde{P}_{m-1}\left(I-\tilde{P}_{m}\right)+\tilde{P}_{m},
$$

for all $z \in \mathbb{D}^{n}$. Thus

$$
\tilde{\Theta}(\boldsymbol{z})=\tilde{\Theta}_{1}(\boldsymbol{z})\left(I-\tilde{P}_{2}\right) \cdots\left(I-\tilde{P}_{m}\right)+\tilde{P}_{2}\left(I-\tilde{P}_{3}\right) \cdots\left(I-\tilde{P}_{m}\right)+\cdots+\tilde{P}_{m-1}\left(I-\tilde{P}_{m}\right)+\tilde{P}_{m},
$$

where $\tilde{\Theta}_{1}(\boldsymbol{z})=\Theta_{1}\left(z_{1}\right)$ for all $\boldsymbol{z} \in \mathbb{D}^{n}$. We therefore have

$$
M_{\tilde{\Theta}} M_{\tilde{\Theta}}^{*}=\tilde{P}_{1}\left(I-\tilde{P}_{2}\right) \cdots\left(I-\tilde{P}_{m}\right)+\tilde{P}_{2}\left(I-\tilde{P}_{3}\right) \cdots\left(I-\tilde{P}_{m}\right)+\cdots+\tilde{P}_{m-1}\left(I-\tilde{P}_{m}\right)+\tilde{P}_{m}
$$

where $\tilde{P}_{1}=M_{\tilde{\Theta}_{1}} M_{\tilde{\Theta}_{1}}^{*}$. Consequently,

$$
M_{\tilde{\Theta}} M_{\tilde{\Theta}}^{*}=I_{H^{2}\left(\mathbb{D}^{n}\right)}-\prod_{i=1}^{m}\left(I_{H^{2}\left(\mathbb{D}^{n}\right)}-\tilde{P}_{i}\right)
$$


and hence

$$
I_{H^{2}\left(\mathbb{D}^{n}\right)}-M_{\tilde{\Theta}} M_{\tilde{\Theta}}^{*}=\prod_{i=1}^{m}\left(I_{H^{2}\left(\mathbb{D}^{n}\right)}-\tilde{P}_{i}\right) .
$$

Therefore, we conclude that

$$
\left(\operatorname{ran} M_{\Theta}\right)^{\perp}=\underbrace{\left(\Theta_{1} H^{2}(\mathbb{D})\right)^{\perp} \otimes\left(P_{2} H^{2}(\mathbb{D})\right)^{\perp} \otimes \cdots \otimes\left(P_{m} H^{2}(\mathbb{D})\right)^{\perp}}_{m \text { times }} \otimes \underbrace{H^{2}(\mathbb{D}) \otimes \cdots \otimes H^{2}(\mathbb{D})}_{(n-m) \text { times }} .
$$

Combine this with the assumption that $\left(\operatorname{ran} M_{\Theta}\right)^{\perp}$ is a quotient module of $H^{2}\left(\mathbb{D}^{n}\right)$ to conclude that $\left(\operatorname{ran} M_{\Theta}\right)^{\perp}$ is a doubly commuting quotient module. This completes the proof.

The above result is a several variables generalization $(n \geq 2)$ of Theorem 3.1 in [25] by Qin and Yang.

\section{Rigidity of Submodules}

Let $\mathcal{M}_{i} \subseteq H^{2}\left(\mathbb{D}^{n}\right), i=1,2$, be two submodules of $H^{2}\left(\mathbb{D}^{n}\right)$. We say that $\mathcal{S}_{1}$ and $\mathcal{S}_{2}$ are unitarily equivalent if there exists a unitary map $U: \mathcal{S}_{1} \rightarrow \mathcal{S}_{2}$ such that

$$
U\left(\left.M_{z_{i}}\right|_{\mathcal{S}_{1}}\right)=\left(\left.M_{z_{i}}\right|_{\mathcal{S}_{2}}\right) U
$$

or equivalently,

$$
U M_{z_{i}}=M_{z_{i}} U
$$

for all $i=1, \ldots, n$.

A consequence of Beurling's theorem ensures that, any pair of non-zero submodules of $H^{2}(\mathbb{D})$ are unitarily equivalent. The conclusion also follows directly from the unitary invariance property of the index of the wandering subspaces associated with the shift operators. This phenomenon is subtle, and in general not true for many other Hilbert modules. For instance, a pair of submodules $\mathcal{S}_{1}$ and $\mathcal{S}_{2}$ of the Bergman modules $L_{a}^{2}\left(\mathbb{B}^{n}\right)$ are unitarily equivalent if and only if $\mathcal{S}_{1}=\mathcal{S}_{2}$ (see [26], [24]). We refer the reader to [12], [13], [14], [15], [29] and [19] for more results on the rigidity of submodules and quotient modules of Hilbert modules over domains in $\mathbb{C}^{n}$.

The submodules corresponding to the doubly commuting quotient modules also holds the rigidity property. This is essentially a particular case of a rigidity result due to Agrawal, Clark and Douglas (Corollary 4 in [2]. See also [21]).

Theorem 4.1. (Agrawal, Clark and Douglas) Let $\mathcal{S}_{1}$ and $\mathcal{S}_{2}$ be two submodules of $H^{2}\left(\mathbb{D}^{n}\right)$, both of which contain functions independent of $z_{i}$ for $i=1, \ldots, n$. Then $\mathcal{S}_{1}$ and $\mathcal{S}_{2}$ are unitarily equivalent if and only if they are equal.

In particular, we obtain a generalization of the rigidity theorem for $n=2$ (see Corollary 2.3 in [30]).

Corollary 4.2. Let $\mathcal{S}_{\Theta}=\sum_{i=1}^{n} \tilde{\Theta}_{i} H^{2}(\mathbb{D})^{n}$ and $\mathcal{S}_{\Phi}=\sum_{i=1}^{n} \tilde{\Phi}_{i} H^{2}(\mathbb{D})^{n}$ be a pair of submodules of $H^{2}(\mathbb{D})^{n}$, where $\tilde{\Theta}_{i}(\boldsymbol{z})=\Theta_{i}\left(z_{i}\right)$ and $\tilde{\Phi}_{i}(\boldsymbol{z})=\Phi_{i}\left(z_{i}\right)$ for inner functions $\Theta_{i}, \Phi_{i} \in H^{\infty}(\mathbb{D})$ and $\boldsymbol{z} \in \mathbb{D}^{n}$ and $i=1, \ldots, n$. Then $\mathcal{S}_{\Theta}$ and $\mathcal{S}_{\Phi}$ are unitarily equivalent if and only if $\mathcal{S}_{\Theta}=\mathcal{S}_{\Phi}$. 
Proof. Clearly $\tilde{\Theta}_{i} \in \mathcal{S}_{\Theta}$ and $\tilde{\Phi}_{i} \in \mathcal{S}_{\Phi}$ are independent of $\left\{z_{1}, \cdots, z_{i-1}, z_{i+1}, \ldots, z_{n}\right\}$ for all $i=1, \ldots, n$. Therefore, the submodules $\mathcal{S}_{\Theta}$ and $\mathcal{S}_{\Phi}$ contains functions independent of $z_{i}$ for all $i=1, \ldots, n$. Consequently, if $\mathcal{S}_{\Phi}$ and $\mathcal{S}_{\Phi}$ are unitarily equivalent then $\mathcal{S}_{\Theta}=\mathcal{S}_{\Phi}$.

The following result is a generalization of Corollary 4.4 in [30] and is a consequence of the rigidity result.

Corollary 4.3. Let $\mathcal{S}_{\Theta}=\sum_{i=1}^{n} \tilde{\Theta}_{i} H^{2}(\mathbb{D})^{n}$ be a submodules of $H^{2}(\mathbb{D})^{n}$, where $\tilde{\Theta}_{i}(\boldsymbol{z})=\Theta_{i}\left(z_{i}\right)$ for inner functions $\Theta_{i} \in H^{\infty}(\mathbb{D})$ for all $i=1, \ldots, n$ and $\boldsymbol{z} \in \mathbb{D}^{n}$. Then $\mathcal{S}_{\Theta}$ and $H^{2}\left(\mathbb{D}^{n}\right)$ are not unitarily equivalent.

Proof. The result follows from the previous theorem along with the observation that $\mathcal{S}_{\Theta}^{\perp} \neq$ $\{0\}$.

We close this section by noting that the results above are not true if we drop the assumption that all $\Theta_{i}$ are inner. For instance, if $\Theta_{i}=\Phi_{i}=0$ for all $i \neq 1$ then $\mathcal{S}_{\Theta} \cong \mathcal{S}_{\Phi}$ but in general, $\mathcal{S}_{\Theta} \neq \mathcal{S}_{\Phi}($ see $[22])$.

\section{Concluding REMARKS}

One of the central issues in the study of Hilbert modules is the problem of analyzing essentially normal submodules and quotient modules of a given essentially normal Hilbert module over $\mathbb{C}[\boldsymbol{z}]$. There is, however, a crucial difference between the Hilbert modules of functions defined over the unit ball and the polydisc in $\mathbb{C}^{n}$. For instance, a submodule $\mathcal{S}$ of an essentially normal Hilbert module $\mathcal{H}$ is essentially normal if and only if the quotient module $\mathcal{H} / \mathcal{S}$ is so (see [4], [10]), that is, the study of essentially normal submodules and quotient modules of essentially normal Hilbert modules amounts to the same. However, this is not the case for the study of essentially doubly commuting Hilbert modules over $\mathbb{D}^{n}$. In other words, the theory of essentially doubly commuting submodules and quotient modules of an essentially doubly commuting Hilbert modules are two different concepts.

One could, however, consider the co-doubly commuting submodules as a special class of submodules of the Hardy module and the results of this paper indicates that the general picture of essentially doubly commuting submodules of the Hardy module will by no means be easy to understand (cf. Corollary 2.6). In particular, the homogenous submodules of $H^{2}\left(\mathbb{D}^{2}\right)$ are always essentially doubly commuting [9. Hence Question 1 has an affirmative answer for the class of homogenous submodules of $H^{2}\left(\mathbb{D}^{2}\right)$. It is not known whether the homogeneous submodules of $H^{2}\left(\mathbb{D}^{n}\right)$, when $n \geq 3$, are essentially doubly commuting. Corollary 2.6 gives an indication of a possible answer to the case $n \geq 3$. Results related to essentially normal submodules of the Drury-Arveson module over the unit ball of $\mathbb{C}^{2}$ can be found in [18].

Our result concerning the Beurling-Lax-Halmos inner function, Theorem 3.1, is closely related to the classification theory of multi-isometries (see [7] and [5]) for $n=2$ case. We hope to discuss the general case in a future paper.

We conclude with a result concerning the $C_{0}$ class. Recall that a completely non-unitary contraction $T$ on some Hilbert space $\mathcal{H}$ is said to be in the class $C_{0}$ if there is a non-zero function $\Theta \in H^{\infty}(\mathbb{D})$ such that $\Theta(T)=0$ [23]. 
Proposition 5.1. Let $\mathcal{Q}$ be a non-trivial doubly commuting quotient module of $H^{2}\left(\mathbb{D}^{n}\right)$. Then $R_{z_{i}} \in C_{0}$ for some $1 \leq i \leq n$.

Proof. By virtue of Theorem 2.1, we let $\mathcal{Q}=\mathcal{Q}_{\Theta_{1}} \otimes \cdots \otimes \mathcal{Q}_{\Theta_{n}}$ and $\mathcal{Q}_{\Theta_{i}} \neq H^{2}(\mathbb{D})$ for some $1 \leq i \leq n$. Consequently, $\Theta_{i}\left(R_{z}\right)=0$ and hence

$$
\tilde{\Theta}_{i}\left(R_{z_{i}}\right)=I_{\mathcal{Q}_{\Theta_{1}}} \otimes \cdots \otimes \underbrace{\Theta_{i}\left(R_{z}\right)}_{i^{t h}} \otimes \cdots \otimes I_{\mathcal{Q}_{\Theta_{n}}}=0 .
$$

This concludes the proof.

The above result for the case $n=2$ is due to Douglas and Yang (see Proposition 4.1 in [11]). However, our proof is more elementary.

\section{REFERENCES}

[1] P. Ahern and D. Clark, Invariant subspaces and analytic continuation in several variables, J. Math. Mech. $19(1969 / 1970)$ 963-969.

[2] O. Agrawal, D. Clark and R. Douglas, Invariant subspaces in the polydisk, Pacific J. Math. 121(1986), $1-11$.

[3] W. Arveson, Quotients of standard Hilbert modules, Trans. Amer. Math. Soc. 359 (2007), no. 12, 60276055 .

[4] W. Arveson, p-summable commutators in dimension d, J. Operator Theory 54 (2005), no. 1, 101-117.

[5] H. Bercovici, R. Douglas and C. Foias, On the classification of multi-isometries, Acta Sci. Math. (Szeged) 72 (2006), no. 3-4, 639-661.

[6] H. Bercovici, Operator theory and arithmetic in $H^{\infty}$, Mathematical Surveys and Monographs, No. 26, A.M.S., Providence, Rhode Island, 1988.

[7] C. Berger, L. Coburn and A. Lebow, Representation and index theory for $C^{*}$-algebras generated by commuting isometries, J. Funct. Anal. 27 (1978), 51-99.

[8] A. Beurling, On two problems concerning linear transformations in Hilbert space, Acta Math. 81 (1949), 239-255.

[9] R. Curto, P. Muhly and K. Yan, The $C^{*}$-algebra of an homogeneous ideal in two variables is type I, Current topics in operator algebras (Nara, 1990), World Sci. Publ., River Edge, NJ, 1991, 130-136.

[10] R. Douglas, Essentially reductive Hilbert modules, J. Operator Theory 55 (2006), no. 1, 117-133.

[11] R. Douglas and R. Yang, Operator theory in the Hardy space over the bidisk. I, Integral Equations Operator Theory, 38(2000), 207-221.

[12] R. Douglas and R. Yang, Quotient Hardy modules, Houston J. Math. 24 (1998), no. 3, $507-517$.

[13] R. Douglas, V. Paulsen, C. Sah and K. Yan, Algebraic reduction and rigidity for Hilbert modules, Amer. J. Math. 117 (1995), 75-92.

[14] R. Douglas and C. Foias, Uniqueness of multi-variate canonical models, Acta Sci. Math. (Szeged) 57 (1993), no. 1-4, 79-81.

[15] R. Douglas and K. Yan, On the rigidity of Hardy submodules, Integral Equations Operator Theory, 13 (1990), 350-363.

[16] R. Douglas and V. Paulsen, Hilbert Modules over Function Algebras, Research Notes in Mathematics Series, 47, Longman, Harlow, 1989.

[17] C. Fefferman and E. Stein, $H^{p}$ spaces of several variables, Acta Math. 129 (1972), no. 3-4, 137-193.

[18] K. Guo, Defect operators for submodules of $H_{d}^{2}$, J. Reine Angew. Math. 573 (2004), 181-209.

[19] K. Guo, Equivalence of Hardy submodules generated by polynomials, J. Funct. Anal. 178 (2000), $343-371$.

[20] K. Izuchi, T. Nakazi and M. Seto, Backward shift invariant subspaces in the bidisc II, J. Oper. Theory 51 (2004), 361-376. 
[21] K. Izuchi, Unitary equivalence of invariant subspaces in the polydisk, Pacific J. Math. 130 (1987), no. 2, 351-358.

[22] V. Mandrekar, The validity of Beurling theorems in polydiscs, Proc. Amer. Math. Soc. 103 (1988), 145148.

[23] B. Sz.-Nagy and C. Foias, Harmonic Analysis of Operators on Hilbert Space, North Holland, Amsterdam, 1970.

[24] M. Putinar, On the rigidity of Bergman submodules, Amer. J. Math. 116 (1994), 1421-1432.

[25] Y. Qin and R. Yang, A characterization of submodules via Beurling-Lax-Halmos theorem, To appera in Proc. of AMS.

[26] S. Richter, Unitary equivalence of invariant subspaces of Bergman and Dirichlet spaces, Pac. J. Math. 133 (1988), 151-156.

[27] W. Rudin, Function Theory in Polydiscs, Benjamin, New York 1969.

[28] J. Sarkar, Jordan blocks of $H^{2}\left(\mathbb{D}^{n}\right)$, arXiv:1303.1041, to appear in Journal of Operator theory.

[29] R. Yang, On two variable Jordan block. II, Integral Equations Operator Theory 56 (2006), no. 3, 431-449.

[30] R. Yang, Hilbert-Schmidt submodules and issues of unitary equivalence, J. Operator Theory 53 (2005), no. $1,169-184$.

Indian Statistical Institute, Statistics and Mathematics Unit, 8th Mile, Mysore Road, RVCE Post, Bangalore, 560059, India

E-mail address: jay@isibang.ac.in, jaydeb@gmail.com 